\title{
NSCs are permissive to oncolytic Myxoma virus and provide a delivery method for targeted ovarian cancer therapy
}

\author{
Yvonne Cornejo ${ }^{1,2}$, Min Li ${ }^{3}$, Thanh H. Dellinger ${ }^{4}$, Rachael Mooney ${ }^{1}$, Masmudur M. \\ Rahman $^{5}$, Grant McFadden ${ }^{5}$, Karen S. Aboody ${ }^{1,6}$ and Mohamed Hammad ${ }^{1}$ \\ ${ }^{1}$ Department of Stem Cell \& Developmental Biology, City of Hope, Duarte, CA 91010, USA \\ ${ }^{2}$ Irell \& Manella Graduate School for Biological Sciences, Beckman Research Institute, City of Hope, Duarte, CA 91010, USA \\ ${ }^{3}$ Department of Information Sciences, Division of Biostatistics at the Beckman Research Institute, City of Hope, Duarte, CA \\ 91010, USA \\ ${ }^{4}$ Division of Gynecologic Surgery, Department of Surgery, City of Hope, CA 91010, USA \\ ${ }^{5}$ Biodesign Institute, Arizona State University, Tempe, AZ 85281, USA \\ ${ }^{6}$ Division of Neurosurgery, City of Hope, Duarte, CA 91010, USA \\ Correspondence to: Mohamed Hammad, email: mhammad@coh.org
}

Keywords: oncolytic virotherapy; myxoma; NSCs; ovarian cancer

Received: October 09, $2020 \quad$ Accepted: December 03, $2020 \quad$ Published: December 22, 2020

Copyright: (c) 2020 Cornejo et al. This is an open access article distributed under the terms of the Creative Commons Attribution License (CC BY 3.0), which permits unrestricted use, distribution, and reproduction in any medium, provided the original author and source are credited.

\section{ABSTRACT}

Despite the development of many anticancer agents over the past 20 years, ovarian cancer remains the most lethal gynecologic malignancy. Due to a lack of effective screening, the majority of patients with ovarian cancer are diagnosed at an advanced stage, and only $\sim 20 \%$ of patients are cured. Thus, in addition to improved screening methods, there is an urgent need for novel anticancer agents that are effective against late-stage, metastatic disease. Oncolytic virotherapy is a promising approach; unfortunately, systemic delivery of viruses to tumors remains a major challenge. In this regard, neural stem/progenitor cells (NSCs) with wellestablished tumor-homing properties may serve as an effective delivery platform for oncolytic viruses. In this study, we tested the efficacy of myxoma virus (MYXV), a rabbit-specific poxvirus that has demonstrated efficacy against a variety of tumors, using human and mouse ovarian cancer cell lines. We showed that MYXV effectively lysed ovarian cancer cells in vitro, reducing their viability. We also demonstrated that MYXV can infect human NSCs, specifically the clonal HB1.F3.CD21 NSC line. Taken together, these results suggest that NSC-mediated delivery of MYXV may be a promising strategy for achieving more selectively targeted anti-tumor efficacy.

\section{INTRODUCTION}

Chemotherapy and surgery have improved greatly over the last several years, however, ovarian cancer remains lethal with over 21,000 women in the United States expected to be newly diagnosed and almost 14,000 deaths expected this year alone [1-3]. One of the major limitations to effectively treating ovarian cancer is that most patients are diagnosed only after their primary tumor metastasizes to the abdomen and by that time their five-year survival rate is about $45 \%$ after Standard Of Care (SOC) treatment [4]. Therefore, it is important to develop new therapies that target metastatic lesions, as well as the primary tumor.
One promising strategy is the use of oncolytic viruses (OVs). These viruses take advantage of faulty mechanisms or pathways commonly found in tumor cells, which allows them to infect and replicate within the tumor cells while sparing healthy cells $[5,6]$. The viruses are then able to lyse the tumor cells, irrespective of chemoresistance, and continue infecting neighboring tumor cells to amplify their anti-neoplastic effects. Once the virus reaches normal tissue, no further replication occurs [7]. Additionally, since new tumor antigens are exposed upon lysis, OVs are able to stimulate immune recognition of the cancer cells, further enhancing their therapeutic efficacy $[8,9]$. 
Myxoma virus (MYXV), a rabbit-specific poxvirus, has recently been considered for use in oncolytic virotherapy [10-12]. Although it is specific to the European rabbit (causing a lethal disease called myxomatosis) and does not replicate in any non-rabbit normal host cells, it has displayed tumoricidal effect against many human tumor cells [13]. This is thought to occur due to cancer cells: 1) failure to produce appropriate anti-viral responses that can efficiently stop MYXV replication; and 2) constitutive upregulation of pathways associated with cellular transformation that support MYXV replication [14-16].

One major limitation in using OVs to treat cancer is that free viruses can be neutralized by antibodies present in the host patient (due to pre-existing viral immunity), making repeat administration difficult [17]. One way to overcome this limitation is to load the OVs into neural stem/progenitor cells (NSCs). NSCs are tumor-tropic, enabling selective delivery of viruses to tumor cells, minimizing off-target effects. In addition to promoting more effective, localized infection this delivery platform protects the virus from neutralization, thereby increasing viral distribution at tumor sites. We pursued in vitro studies in the present study to investigate the pre-clinical efficacy of NSC delivered MYXV on ovarian cancer cells.

\section{RESULTS}

\section{MYXV showed oncolytic effect for ovarian cancer cells}

We incubated human (OVCAR8 and SKOV3) and murine (ID8) ovarian cancer cells with MYXV expressing fluorescence reporter proteins GFP (representative of infection) and $\operatorname{Td} \operatorname{Tr}$ (representative of replication) (vMyxGFP-TdTomato). These ovarian cancer cells were infected at a multiplicity of infection (MOI) of 10 and time-lapse images captured every $8 \mathrm{~h}$ for $48 \mathrm{~h}$ post-infection.

Cells that were not infected were used as the negative control. Total areas $\left(\mu \mathrm{m}^{2} /\right.$ well) of GFP and RFP were calculated and analyzed. All cell lines showed a significant increase in both infection (Figure 1A-1C) and replication (Figure 1D-1F) over time. Next, we infected the same cancer cell lines with vMyx-GFP-TdTr at multiple MOIs and pursued MTS assays to assess the viability of the cells two days after infection. We observed that all cell lines infected with vMyx-GFP-TdTr at MOIs of 3-10 have shown reduced viability compared to noninfected ones (Figure 1G-1I). These data show that vMyxGFP-TdTr can infect, replicate in, and kill ovarian cancer cell lines.

\section{NSCs can be infected with vMyx-GFP-TdTr}

In the present study, we used HB1.F3.CD21 NSCs to package MYXV. Specifically, we infected the NSCs with vMyx-GFP-TdTr at an MOI of 10 and captured time- lapse images every $8 \mathrm{~h}$ for $48 \mathrm{~h}$ post-infection, as we did for the ovarian cancer cell lines. The NSCs showed a significant increase in infection and replication over time (Figure 2A and 2B). We also assessed the effect of vMyxGFP-TdTr infection on NSCs viability. Seventy-two $h$ after vMyx-GFP-TdTr infection at an MOI of 1, 50\% of NSCS were still viable (Figure 2C). This is promising given our previous studies demonstrating that NSCs start seeding virus to tumor sites within 1 hour after i.p. injection in ovarian cancer models [26].

\section{DISCUSSION}

Oncolytic virotherapy has been proven to be safe in many studies [18-21]. However, their efficacy in clinical settings was questionable predominantly because of antiviral immunity responses, inadequate delivery of the virus and poor distribution of the virus to tumor sites [21, 22]. Therefore, it is of utmost importance to utilize a wellestablished delivery system to enhance the distribution of viruses to tumors and improve their subsequent clinical outcome. Several groups have delivered viruses using autologous cells, including mesenchymal stem cells (MSCs) [19, 23, 24], CD14+-derived monocytes [23] and irradiated tumor cells, interleukin-2 (IL-2)-expanded $\mathrm{T}$ cells [25]. Though, these approaches are limited by variability in cell expansion potential, cytogenetic stability, and tumor tropism ability and ex vivo viral loading capacity. For example, Mader et al. reported a minimum of two weeks to generate enough amounts of autologous MSCs to treat a patient and around one fifth of the MSC generated showed aberrant karyotypes [24]. MSCs also lose their tumor tropism after 5-6 passages. Hence, we think that using an allogeneic cell line will facilitate clinical translation and long-term scale-up. Furthermore, a cellular delivery vehicle for OVs may considerably advance therapeutic outcomes for treatment regimens that require multiple administrations, especially with the probable following accumulation of neutralizing antibodies against the $\mathrm{OV}$.

With the plausible therapeutic modality of NSC as a delivery platform, we are considering investigating $\mathrm{OV}$ cargo with robust anticancer effect. MYXV is a promising $\mathrm{OV}$, as reports have demonstrated that it can infect established human ovarian tumor cell lines [16].

We formerly confirmed that NSCs migrate to ovarian cancer after intraperitoneal (IP) injection into xenograft mouse models with OVCAR8 or SKOV3 peritoneal metastases $[17,26]$. Hence, we postulate HB1.F3.CD21 NSC line as a reproducible and predictable carrier vehicle for viral delivery and release kinetics [27]. Indeed, we showed that these NSCs enhance delivery of adenovirus to the peritoneal and brain cancers [17, 27].

Our data indicate that MYXV can kill ovarian cancer cells and that NSCs are permissive to MYXV. These results warrant further investigation of the therapeutic 
potential of using an NSC platform to deliver MYXV in vivo, as well as to study their temporal and spatial distribution, intratumoral retainment, and neutralization mediated by the immune system, particularly after multiple rounds of administration [28]. Future studies should also be conducted to determine the extent to which MYXV provokes a secondary anti-tumor immune response following tumor cell lysis. MYXV can also be manipulated to express therapeutic transgenes, a strategy that performed in several OVs $[22,29]$.

\section{MATERIALS AND METHODS}

The human ovarian cancer cell line OVCAR8 was kindly gifted by Dr. Carlotta Glackin (City of Hope). We purchased the human ovarian cancer cell line SKOV3 from
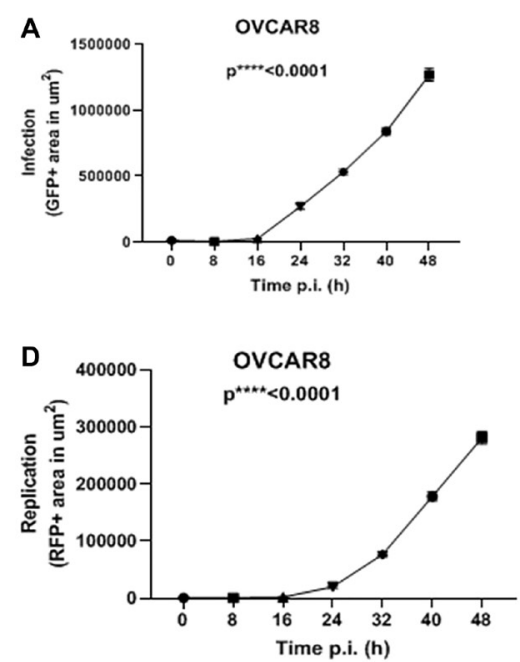

G

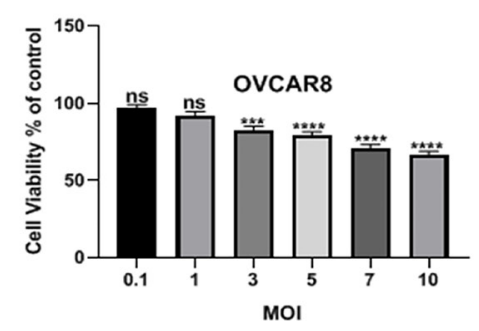

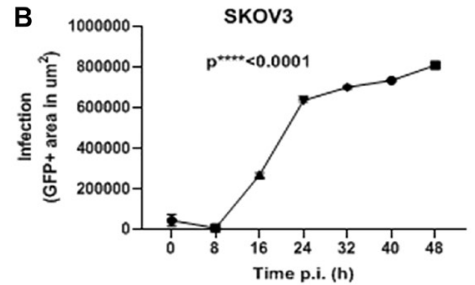
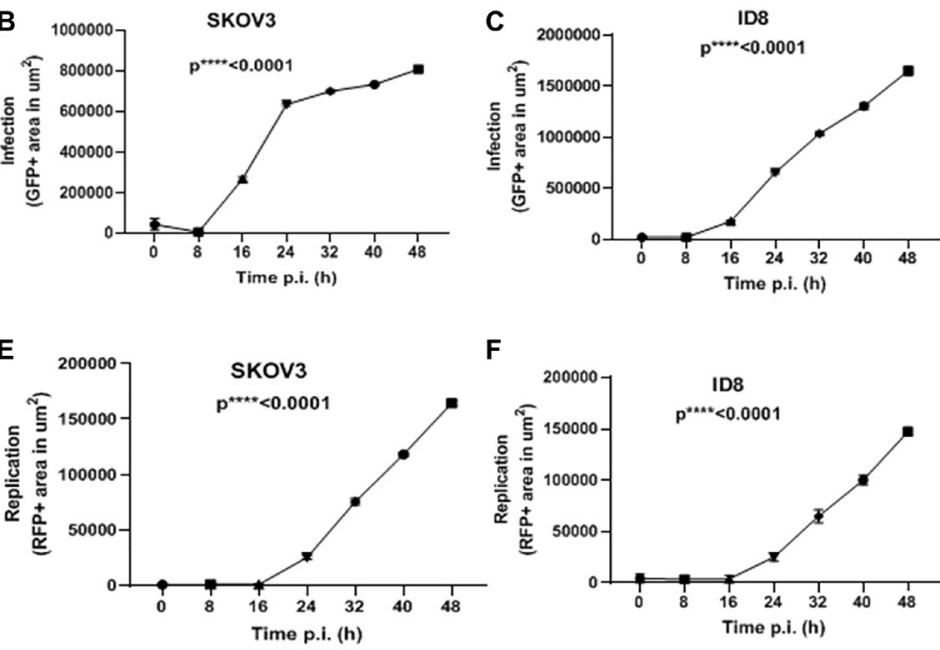

H

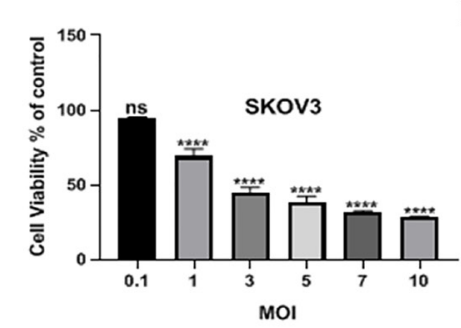

I

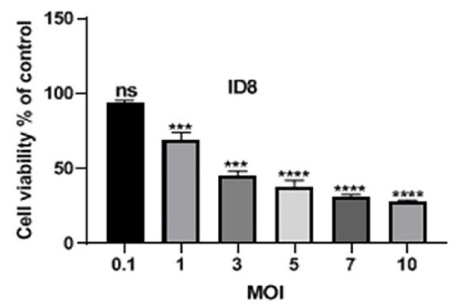

Figure 1: MYXV infects, replicates in, and kills ovarian cancer cells in vitro. (A) OVCAR8, (B) SKOV3, and (C) ID8 ovarian cancer cells were treated with vMyx-GFP-TdTr at an MOI of 10, and infection increased significantly over time post-infection. Similarly, the replication of (D) OVCAR8, (E) SKOV3, and (F) ID8 ovarian cancer cells increased significantly over time following infection. (G) OVCAR8, (H) SKOV3, and (I) ID8 were treated with vMyx-GFP-TdTr at multiple MOIs, and $72 \mathrm{~h}$ later showed significantly lower viability relative to non-infected control cells. Data are shown as mean \pm SEM for at least two repeated experiments. ${ }^{* * *} P<0.001,{ }^{* * * *} P<$ 0.0001, as determined by one-way ANOVA comparing infected vs. non-infected cells. ns, no significant difference.
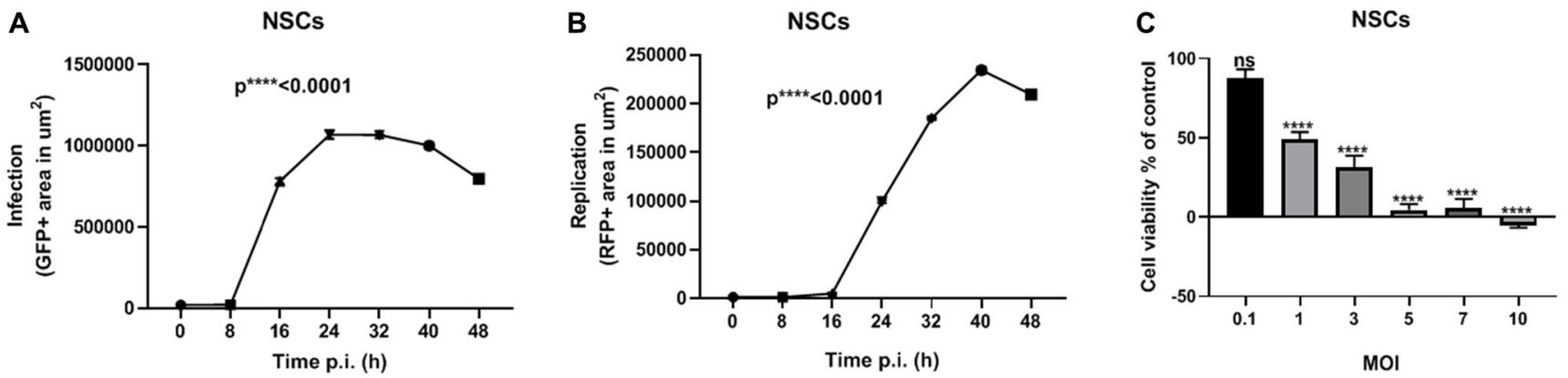

Figure 2: NSCs are permissive to vMyx-GFP-TdTr infection. (A) NSCs were treated with vMyx-GFP-TdTr at an MOI of 10, and infection increased significantly over time post-infection. (B) Similarly, the replication of vMyx-GFP-TdTr increased significantly over time after infection. (C) NSCs were infected with vMyx-GFP-TdTr at various MOIs, and $72 \mathrm{~h}$ later showed significantly lower viability relative to non-infected control cells. Data are shown as mean $\pm \mathrm{SEM}$ for at least two repeated experiments. ${ }^{* * * *} P<0.0001$, as determined by one-way ANOVA comparing infected vs. non-infected cells. ns, no significant difference. 
ATCC. Dr. Katherine Roby (University of Kansas) provided us with the murine ovarian line ID8 then we transduced it to express firefly luciferase. We cultured the ovarian cancer cell lines in RPMI media supplemented with $1 \%$ penicillinstreptomycin (Invitrogen), 10\% fetal bovine serum (Gemini Bio) and 1\% L-glutamine (Invitrogen). Dr. Seung Kim (University of British Columbia, Canada) [30] provided us with the human, v-myc immortalized HB1.F3.CD NSC line. We used this line to generate NSCs that carry MYXV by infecting them with MYXV at multiple MOIs of for $48 \mathrm{~h}$ in DMEM (Invitrogen). The media was supplemented with 1\% L-glutamine (Invitrogen), 1\% penicillin-streptomycin (Invitrogen) and $10 \%$ fetal bovine serum (Gemini Bio) and incubated at $37^{\circ} \mathrm{C}$ in a humidified incubator (Thermo Electron Corporation) containing $6 \% \mathrm{CO}_{2}$ then harvested. We changed media every 2-3 days and passaged or harvested all cell lines, when they reached confluency at $80 \%$ using EDTA solution (Invitrogen) with $0.25 \%$ trypsin.

MYXV virus used in the current study has been described in previous studies [31, 32] In short, MYXV constructs expressing the enhanced GFP and the tandem dimer (td)-Tomato red fluorescent protein (tdTr) were combined in an intergenic location (between the M135 and M136 genes) to make vMyx-GFP-tdTr. GFP is under the control of a poxvirus early/late synthetic promoter and tdTr is under the control of a poxviral late $(\mathrm{p} 11)$ promoter [15].

Ovarian cancer cells (OVCAR8, SKOV3, and ID8) and NSCs were seeded in 96-well plates (5000/well) and treated with vMyx-GFP-TdTr at an MOI of 10. The 96well plates were placed in an IncuCyte Live-Cell Analysis System (Essen BioScience). Bright field and green and red fluorescence images of the whole field of view for each well were captured every $8 \mathrm{~h}$ for $48 \mathrm{~h}$. Images were visualized, and fluorescent areas/well were calculated using IncuCyte software. In another experiment, ovarian cancer cells and NSCs were seeded and allowed to grow overnight at $37^{\circ} \mathrm{C}$ in an incubator containing $6 \% \mathrm{CO}_{2}$. The next day, vMyx-GFP-TdTomato was added to each well at various MOIs $(0.1,1,3,5,7$ and 10$)$ in $100 \mu \mathrm{L}$. MTS assay was conducted as previously discussed [21].

Unless otherwise stated, data are presented as means \pm standard error of mean (SEM). One-way ANOVA was used compare virus fluorescence at each time point among the groups with ${ }^{*} P<0.05$ considered to be significant.

\section{Abbreviations}

IP: Intraperitoneal; MOI: multiplicity of infection; MSC: mesenchymal stem cell; MYXV: Myxoma virus; NSC: neural stem cell; OV: oncolytic virus; PFU: plaqueforming unit.

\section{Author contributions}

Conceptualization, K.S.A., G.M., M.H., T.D.; Methodology, M.H., Y.C.; Formal Analysis, M.H., M.L.;
Investigation, M.H., Y.C.; Writing-Original Draft, M.H.; Writing-Review \& Editing, M.H., K.S.A., G.M., R.M.; Visualization: M.H., Y.C., K.S.A.; Funding Acquisition, G.M., K.S.A.; Resources, G.M., K.S.A.; Supervision, K.S.A., G.M.

\section{ACKNOWLEDGMENTS}

We are grateful to Kerin Higa, Ph.D., for editing the manuscript.

\section{CONFLICTS OF INTEREST}

K.S.A. is a board member and shareholder of TheraBiologics, Inc., M.M.R. is consultant and G.M. is co-founder and shareholder of OncoMyx Therapeutics, a biotech company devoted to the development of multiarmed MYXV for treatment of cancer. G.M. is a founder and a research advisor director of Oncomyx, a company that is developing oncolytic immunotherapies with an unambiguous, promotable advantage that eradicate hard-to-cure cancers. All other authors declare no competing interests. The funders had no role in the design of the study; in the collection, analyses, or interpretation of data; in the writing of the manuscript, or in the decision to publish the results.

\section{FUNDING}

This work was funded by STOP CANCER, the Rosalinde and Arthur Gilbert Foundation, the California Institute of Regenerative Medicine, the Alvarez Family Foundation, the Anthony F. \& Susan M. Markel Foundation, the Ben and Catherine Ivy Foundation, City of Hope, and National Cancer Institute grants R43CA86768, R44CA8678, and R01CA197359. Research reported in this publication includes work performed by M.L. in the City of Hope Biostatistics Core, which is supported by the National Cancer Institute of the NIH under award number P30CA033572. The content is solely the responsibility of the authors and does not necessarily represent the official views of the NIH. Materials Transfer Information is available from the City of Hope Office of Technology Licensing.

\section{REFERENCES}

1. Siegel R, Naishadham D, Jemal A. Cancer statistics, 2013. CA Cancer J Clin. 2013; 63:11-30. https://doi.org/10.3322/ caac.21166. [PubMed]

2. Znaor A, van den Hurk C, Primic-Zakelj M, Agius D, Coza D, Demetriou A, Dimitrova N, Eser S, Karakilinc H, Zivkovic S, Bray F, Coebergh JW. Cancer incidence and mortality patterns in South Eastern Europe in the last decade: gaps persist compared with the rest of Europe. Eur J Cancer. 2013; 49:1683-91. https://doi.org/10.1016/j. ejca.2012.11.030. [PubMed] 
3. Bray F, Ren JS, Masuyer E, Ferlay J. Global estimates of cancer prevalence for 27 sites in the adult population in 2008. Int J Cancer. 2013; 132:1133-45. https://doi. org/10.1002/ijc.27711. [ [PubMed]

4. Webb PM, Jordan SJ. Epidemiology of epithelial ovarian cancer. Best Pract Res Clin Obstet Gynaecol. 2017; 41:3-14. https://doi.org/10.1016/j.bpobgyn.2016.08.006. [PubMed]

5. Correa RJ, Komar M, Tong JG, Sivapragasam M, Rahman MM, McFadden G, Dimattia GE, Shepherd TG. Myxoma virus-mediated oncolysis of ascites-derived human ovarian cancer cells and spheroids is impacted by differential AKT activity. Gynecol Oncol. 2012; 125:441-50. https://doi. org/10.1016/i.ygyno.2012.01.048. [PubMed]

6. Ulasov IV, Zhu ZB, Tyler MA, Han Y, Rivera AA, Khramtsov A, Curiel DT, Lesniak MS. Survivin-driven and fiber-modified oncolytic adenovirus exhibits potent antitumor activity in established intracranial glioma. Hum Gene Ther. 2007; 18:589-602. https://doi.org/10.1089/ hum.2007.002. [PubMed]

7. Kim J, Hall RR, Lesniak MS, Ahmed AU. Stem CellBased Cell Carrier for Targeted Oncolytic Virotherapy: Translational Opportunity and Open Questions. Viruses. 2015; 7:6200-17. https://doi.org/10.3390/v7122921. [PubMed]

8. Ding J. Oncolytic virus as a cancer stem cell killer: progress and challenges. Stem Cell Investig. 2014; 1:22. https://doi. org/10.3978/j.issn.2306-9759.2014.12.02. [PubMed]

9. Nakamori M, Fu X, Meng F, Jin A, Tao L, Bast RC Jr, Zhang X. Effective therapy of metastatic ovarian cancer with an oncolytic herpes simplex virus incorporating two membrane fusion mechanisms. Clin Cancer Res. 2003; 9:2727-33. [PubMed]

10. Stanford MM, McFadden G. Myxoma virus and oncolytic virotherapy: a new biologic weapon in the war against cancer. Expert Opin Biol Ther. 2007; 7:1415-25. https:// doi.org/10.1517/14712598.7.9.1415. [PubMed]

11. Rahman MM, McFadden G. Oncolytic Virotherapy with Myxoma Virus. J Clin Med. 2020; 9:21. https://doi. org/10.3390/jcm9010171. [PubMed]

12. Chan WM, Rahman MM, McFadden G. Oncolytic myxoma virus: the path to clinic. Vaccine. 2013; 31:4252-8. https:// doi.org/10.1016/j.vaccine.2013.05.056. [PubMed]

13. Sypula J, Wang F, Ma Y, Bell J, McFadden G. Myxoma virus tropism in human tumor cells. Gene Ther Mol Biol. 2004; 8:103-14.

14. Bartee E, McFadden G. Human cancer cells have specifically lost the ability to induce the synergistic state caused by tumor necrosis factor plus interferon-beta. Cytokine. 2009; 47:199-205. https://doi.org/10.1016/j. cyto.2009.06.006. [PubMed]

15. Bartee E, Mohamed MR, Lopez MC, Baker HV, McFadden $\mathrm{G}$. The addition of tumor necrosis factor plus beta interferon induces a novel synergistic antiviral state against poxviruses in primary human fibroblasts. J Virol. 2009; 83:498-511. https://doi.org/10.1128/JVI.01376-08. [PubMed]

16. Wang G, Barrett JW, Stanford M, Werden SJ, Johnston JB, Gao X, Sun M, Cheng JQ, McFadden G. Infection of human cancer cells with myxoma virus requires Akt activation via interaction with a viral ankyrin-repeat host range factor. Proc Natl Acad Sci U S A. 2006; 103:4640-5. https://doi. org/10.1073/pnas.0509341103. [PubMed]

17. Mooney R, Majid AA, Batalla-Covello J, Machado D, Liu X, Gonzaga J, Tirughana R, Hammad M, Lesniak MS, Curiel DT, Aboody KS. Enhanced Delivery of Oncolytic Adenovirus by Neural Stem Cells for Treatment of Metastatic Ovarian Cancer. Mol Ther Oncolytics. 2019; 12:79-92. h https://doi.org/10.1016/j.omto.2018.12.003. [PubMed]

18. Cannistra SA. Cancer of the ovary. N Engl J Med. 2004; 351:2519-29. https://doi.org/10.1056/NEJMra041842. [PubMed]

19. Li S, Tong J, Rahman MM, Shepherd TG, McFadden G. Oncolytic virotherapy for ovarian cancer. Oncolytic Virother. 2012; 1:1-21. https://doi.org/10.2147/ov.s31626. [PubMed]

20. Galanis E. Therapeutic potential of oncolytic measles virus: promises and challenges. Clin Pharmacol Ther. 2010; 88:620-5. https://doi.org/10.1038/clpt.2010.211. [PubMed]

21. Hammad M, Cornejo YR, Batalla-Covello J, Majid AA, Burke C, Liu Z, Yuan YC, Li M, Dellinger TH, Lu J, Chen NG, Fong Y, Aboody KS, et al. Neural Stem Cells Improve the Delivery of Oncolytic Chimeric Orthopoxvirus in a Metastatic Ovarian Cancer Model. Mol Ther Oncolytics. 2020; 18:326-34. https://doi.org/10.1016/j. omto.2020.07.002. [PubMed]

22. Zheng M, Huang J, Tong A, Yang H. Oncolytic Viruses for Cancer Therapy: Barriers and Recent Advances. Mol Ther Oncolytics. 2019; 15:234-47. https://doi.org/10.1016/j. omto.2019.10.007. [PubMed]

23. Peng KW, Dogan A, Vrana J, Liu C, Ong HT, Kumar S, Dispenzieri A, Dietz AB, Russell SJ. Tumor-associated macrophages infiltrate plasmacytomas and can serve as cell carriers for oncolytic measles virotherapy of disseminated myeloma. Am J Hematol. 2009; 84:401-7. https://doi. org/10.1002/ajh.21444. [PubMed]

24. Mader EK, Butler G, Dowdy SC, Mariani A, Knutson KL, Federspiel MJ, Russell SJ, Galanis E, Dietz AB, Peng KW. Optimizing patient derived mesenchymal stem cells as virus carriers for a phase I clinical trial in ovarian cancer. J Transl Med. 2013; 11:20. https://doi.org/10.1186/1479-5876-1120. [PubMed]

25. Ong HT, Hasegawa K, Dietz AB, Russell SJ, Peng KW. Evaluation of $\mathrm{T}$ cells as carriers for systemic measles virotherapy in the presence of antiviral antibodies. Gene Ther. 2007; 14:324-33. https://doi.org/10.1038/ sj.gt.3302880. [PubMed]

26. Cao P, Mooney R, Tirughana R, Abidi W, Aramburo S, Flores L, Gilchrist M, Nwokafor U, Haber T, Tiet P, Annala AJ, Han 
E, Dellinger T, et al. Intraperitoneal Administration of Neural Stem Cell-Nanoparticle Conjugates Targets Chemotherapy to Ovarian Tumors. Bioconjug Chem. 2017; 28:1767-76. https://doi.org/10.1021/acs.bioconjchem.7b00237. [PubMed]

27. Ahmed AU, Thaci B, Alexiades NG, Han Y, Qian S, Liu F, Balyasnikova IV, Ulasov IY, Aboody KS, Lesniak MS. Neural stem cell-based cell carriers enhance therapeutic efficacy of an oncolytic adenovirus in an orthotopic mouse model of human glioblastoma. Mol Ther. 2011; 19:1714 26. https://doi.org/10.1038/mt.2011.100. [PubMed]

28. Shmeleva EV, Smith GL, Ferguson BJ. Enhanced Efficacy of Vaccination With Vaccinia Virus in Old vs. Young Mice. Front Immunol. 2019; 10:1780. https://doi.org/10.3389/ fimmu.2019.01780. [PubMed]

29. Grossardt C, Engeland CE, Bossow S, Halama N, Zaoui K, Leber MF, Springfeld C, Jaeger D, von Kalle C, Ungerechts G. Granulocyte-macrophage colony-stimulating factorarmed oncolytic measles virus is an effective therapeutic cancer vaccine. Hum Gene Ther. 2013; 24:644-54. https:// doi.org/10.1089/hum.2012.205. [PubMed]
30. Kendall SE, Najbauer J, Johnston HF, Metz MZ, Li S, Bowers M, Garcia E, Kim SU, Barish ME, Aboody KS, Glackin CA. Neural stem cell targeting of glioma is dependent on phosphoinositide 3-kinase signaling. Stem Cells. 2008; 26:1575-86. https://doi.org/10.1634/ stemcells.2007-0887. [PubMed]

31. Liu J, Wennier S, Reinhard M, Roy E, MacNeill A, McFadden G. Myxoma virus expressing interleukin-15 fails to cause lethal myxomatosis in European rabbits. J Virol. 2009; 83:5933-8. https://doi.org/10.1128/JVI.00204-09. [PubMed]

32. Johnston JB, Barrett JW, Chang W, Chung CS, Zeng W, Masters J, Mann M, Wang F, Cao J, McFadden G. Role of the serine-threonine kinase PAK-1 in myxoma virus replication. J Virol. 2003; 77:5877-88. https://doi. org/10.1128/jvi.77.10.5877-5888.2003. [PubMed] 